\title{
PARAGUAYOS EN ARGENTINA: PROPENSIÓN A EMIGRAR Y CARACTERÍSTICAS SOCIODEMOGRÁFICAS (2001-2010)
}

\author{
Paraguayans in Argentina: Propensity to emigrate and sociodemographic \\ characteristics (2001-2010)
}

Víctor E. Torres*

\section{Resumen}

Este trabajo se refiere a las principales características de la comunidad paraguaya en Argentina según los censos relevados desde 1869, pero con particular énfasis en los de 2001 y 2010 con el objetivo de tratar con mayor profundidad el panorama actual. Además, se presenta un modelo de regresión logística binaria que estima la propensión a emigrar desde Paraguay hacia Argentina. Los resultados señalan que el odds de emigrar es mayor en las personas entre 18 y 30 años de edad, sin empleo, sin hijos y sin unión conyugal. Para ello se utilizan datos obtenidos en una encuesta realizada en Paraguay durante el año 2008.

$$
<\text { Migración }><\text { Paraguay }><\text { Argentina }>
$$

\begin{abstract}
This paper refers to the main characteristics of the paraguayan community living in Argentina, according to censuses carried out since 1869. In order to describe the current features of the process, the analysis is focused on the period between 2001 and 2010. Also, a binary logistic model is presented in order to estimate the propensity to emigrate from Paraguay to Argentina. Results indicate that the odds of emigration are higher in people between 18 and 30 years old, unemployed, single, and with no children. The data used is the one obtained in a survey carried out in Paraguay during 2008.
\end{abstract}

$$
<\text { Migration }><\text { Paraguay }><\text { Argentina }>
$$

\footnotetext{
* Doctor en Demografía, Investigador Asistente CONICET, CIECS - CONICET y UNC - y FCE - UNC. torresedu@gmail.com
} 
Torres. Paraguayos en Argentina: propensión a emigrar y características...

\section{Introducción}

Argentina se ha caracterizado por ser tradicionalmente un país receptor de inmigrantes que llegaron desde ultramar o la propia América Latina, en especial desde los países limítrofes. Al analizar los datos censales, éstos demuestran la importancia que presenta en particular la inmigración paraguaya: desde 1947 es la más numerosa dentro de los países latinoamericanos y a partir del censo 2001 constituye el colectivo extranjero más cuantioso en Argentina.

En la actualidad, según el Censo Nacional de Población, Hogares y Viviendas de Argentina de 2010, la comunidad paraguaya está compuesta por 550.713 personas, manteniéndose como la colectividad extranjera más numerosa pero alcanzando -en valores absolutos- un tamaño máximo nunca antes registrado. Esta cifra representa el 30,5\% del total de inmigrantes en el país, el 1,4\% del total de Argentina (INDEC, 2012) y además equivale aproximadamente al 8,6\% de la población total del Paraguay ${ }^{1}$, superando al 6,2\% de la década previa (Cerrutti y Parrado, 2006).

Al tratarse de un relevante y antiguo fenómeno migratorio, se han realizado numerosos trabajos que revisan sus diversos aspectos; entre ellos están los que abordan la reconstrucción histórica y social del proceso, los cambios producidos en el marco legal y también los que analizan el volumen, razones y evolución de la inmigración paraguaya en Argentina.

Sin embargo, aún es factible indagar sobre la dinámica migratoria paraguaya y una alternativa posible es hacerlo mediante el estudio de factores asociados a la propensión emigratoria. De este modo, el presente trabajo pretende contribuir al estudio de dicha dinámica poniendo en consideración cómo interviene un conjunto de características de los jefes de hogar paraguayos con la toma de decisión de emigrar a Argentina.

El propósito de este trabajo es presentar las principales características sociodemográficas de la colectividad paraguaya correspondientes a la última década. Según los censos nacionales de población de 2001 y 2010, en ese período intercensal hubo un proceso de masculinización de la colectividad paraguaya, ganó peso relativo el grupo de edad comprendido entre 15 y 64 años de edad, mejoró el nivel de escolarización de hombres y mujeres, hay mayor porcentaje de inmigrantes residentes en el Área Metropolitana de Buenos Aires (AMBA) y se incrementó la tasa de actividad tanto en hombres como mujeres.

A continuación, se realiza un análisis de la probabilidad de emigrar empleando variables socioeconómicas de los jefes de hogar de Paraguay: situación conyugal, edad, cantidad de hijos, situación laboral, área de residencia (rural o urbana) y sexo. De esta manera, mediante el análisis de los resultados obtenidos es posible conocer el peso que posee cada una de ellas y así observar la manera en que cada uno de los factores incide en las decisiones migratorias.

${ }^{1}$ Esto si se considera una población total en Paraguay de 6.400.000 personas (según los datos provisorios del Censo 2012 realizado en dicho país, ya que aún no están disponibles los datos definitivos). 
Para el abordaje de lo anterior, se utilizan los datos primarios provistos por la Encuesta de Emigración de Paraguayos, la cual fue realizada en cuatro Departamentos de Paraguay durante el año 2008 y para ello la metodología empleada es un modelo de regresión logística binaria que estima la propensión a emigrar de los jefes de hogar paraguayos. Los resultados indican que esta propensión se incrementa al tratarse de personas menores de 30 años de edad, sin hijos, sin empleo y que no estén casados o unidos. No resultaron estadísticamente significativas las variables sexo ni lugar donde vive (zona rural o urbana).

\section{Principales antecedentes de la inmigración paraguaya en Argentina}

El comienzo de la relación entre Paraguay y Argentina se produjo junto con el inicio de ambas sociedades, durante el siglo XVI, cuando Asunción y Buenos Aires eran provincias hispánicas (Maeder, 2010). Por lo tanto, aunque la afluencia de paraguayos hacia Argentina fue previa al primer censo argentino realizado en 1869, los resultados del mismo señalan la presencia de 3.288 paraguayos. Entre los censos de 1869 y 1895, el tamaño de la colectividad paraguaya creció a un ritmo de 5,9\% promedio anual, alcanzando finalmente un total de 14.562 personas. De éstas, el 69\% (10.023) se encontraba en el Nordeste: 3.962 en Misiones, 1.766 en Formosa, 777 en Chaco y 3.518 en Corrientes. Esto pone de manifiesto la marcada localización paraguaya en las mencionadas provincias a finales del siglo XIX, con predominancia masculina en zonas rurales mientras las mujeres lo hicieron en zonas urbanas.

Otra cualidad distintiva de la comunidad paraguaya de fin de siglo XIX y comienzos del XX fue, a diferencia de la nativa, la mayoritaria cantidad de hombres: en 1914 el índice de masculinidad de paraguayos y argentinos, respectivamente, fue: 132,8 y 88,3 (Corrientes), 149,6 y 123,6 (Chaco), 121,2 y 117,8 (Formosa), 132,7 y 94,4 (Misiones) ${ }^{2,3}$. Si se compara esto con los migrantes de ultramar, éstos últimos registraron valores aún mayores en el índice ya que en el caso de los paraguayos la proximidad territorial con su país de origen permitió la migración en familia o incluso de mujeres solas con mayor frecuencia, resultando así un menor índice de masculinidad que para los europeos (Meichtry y Beck, 1999). Ciertos autores mencionan que en el caso específico de las mujeres paraguayas los casos con índices de masculinidad cercanos a 100 no aluden a una migración por etapas (primero los hombres jefes de hogar y luego sus cónyuges y sus hijos) sino que se tratan de mujeres que migran individualmente (Cacopardo, 2000 y 2002; Cacopardo y Maguid, 2001).

A partir de ese momento y al menos hasta la década de 1950 esta tendencia migratoria se reforzó, registrando la población paraguaya una tasa de crecimiento

\footnotetext{
2 En 1914 Formosa y Misiones concentraron el 46\% de los inmigrantes paraguayos, en Buenos Aires el $11 \%$ y en las otras provincias del país el 43\% restante (Carrón, 1979). Así, la marcada primacía numérica de varones en Formosa y Misiones explica el índice de masculinidad total.

3 En 1895 el índice de masculinidad de paraguayos y argentinos también mostró similar comportamiento. Respectivamente, fue 120,2 y 87,7 en Corrientes; 101,8 y 121,7 en Chaco; 123,8 y 152,8 en Formosa y 124 y 108,4 en Misiones (Meichtry y Beck, 1999).
} 
Torres. Paraguayos en Argentina: propensión a emigrar y características...

promedio anual de 3,6\% en el período 1895-1947. Esto se explica por las oportunidades que surgieron del creciente desarrollo económico argentino, el cual demandó materias primas de la región chaqueña. Pero a mediados del siglo pasado se inició una nueva etapa cuando comenzó la desaceleración de la demanda de productos como el algodón en el Chaco, la yerba mate en Misiones y, por contrapartida, apareció como polo de atracción laboral las grandes ciudades.

Como consecuencia "esto marcó a nivel nacional un manifiesto cambio en las ocupaciones tradicionales de los inmigrantes limítrofes, desde las relacionadas con la explotación forestal y la agricultura para el mercado de consumo interno hasta la industria de la construcción y los servicios domésticos durante el proceso de la industrialización sustitutiva y la nueva etapa de reestructuración económica" (Meichtry y Beck, 1999: 177).

Los cambios mencionados también propiciaron alteraciones en el flujo migratorio: de acuerdo a los datos del censo argentino de 1947 por primera vez los inmigrantes paraguayos constituyeron el grupo más numeroso de extranjeros limítrofes residentes en Argentina (con 93.248 personas) relegando al segundo lugar a Uruguay, el más cuantioso hasta ese momento desde el censo de 1869.

A partir de mediados del siglo XX continuó el ingreso de paraguayos debido a razones políticas y económicas en ambos países. Además, se identifican dos flujos principales: el primero fue consecuencia de la Guerra del Chaco (1936) y de la Guerra Civil paraguaya ocurrida en 1947. Las décadas de dictadura militar en Paraguay provocaron la expulsión de población y al mismo tiempo actuaron como factores de atracción las oportunidades de empleo agrario por el desarrollo de la producción de algodón, caña de azúcar, tabaco y té en el Noreste argentino. Por su parte, el segundo flujo ocurrió desde mediados de los años cincuenta y fue impulsado por motivos económicos, eligiéndose como principal destino el AMBA (Benencia, 2007).

Hacia 1960 se produjo un corrimiento de la estructura etaria de los inmigrantes hacia edades más avanzadas (de 20 a 34 años en lugar de ser concentrado sólo de 20 a 29 años, como ocurrió en ocasión del censo 1895), junto con una mayor presencia femenina particularmente en las edades más jóvenes. De este modo, hubo un doble proceso simultáneo: el incremento de la concentración residencial en el AMBA y la feminización de la migración (Maguid, 1990 y 1997; Marshall, 1983). Para ese entonces comenzó a destacarse la importancia cuantitativa de la colectividad paraguaya: prácticamente el 10\% de la población de dicho país fue censada en Argentina (Benencia, 2009).

En su trabajo, Marshall y Orlansky (1981) destacaron que el gran peso que tuvo la salida de paraguayos al exterior con respecto a la migración interna fue debido no sólo a factores de carácter político sino también a las propias falencias de las estructuras productivas del Paraguay, que no fue capaz de retener el excedente de mano de obra local lo que se tradujo en migración internacional.

Dentro de sus principales características, puede señalarse que el contingente paraguayo fue fundamentalmente de bajo nivel de instrucción. En ese aspecto, Maletta (1992: 8) afirma que: 
En 1970, los datos censales mostraron que sólo un 7,4\% de los inmigrantes paraguayos había cursado 10 o más años de estudios. La mitad de la población paraguaya residente en la Argentina tenía menos de cuatro años de educación formal. Una alta proporción de los residentes paraguayos activos, según los censos, se emplean como obreros de la construcción, peones agrícolas y trabajadoras del servicio doméstico.

Asimismo, el autor menciona la importancia de la migración de retorno y de los trabajadores estacionales, quienes fueron ocupados en la agricultura y en otras tareas relacionadas con la hotelería, el turismo y la gastronomía. Todos ellos permanecían sólo algunos meses en Argentina o por un plazo que oscilaba entre uno y cinco años, con visitas anuales o semestrales a sus lugares de origen.

Pero en la segunda parte de la década de 1970 la cuantía migratoria disminuyó, lo que puede explicarse fundamentalmente por dos causas: la construcción de la represa de Itapú ${ }^{4}$ en la zona oriental de Paraguay (junto con el crecimiento económico general de dicho país) y la contracción económica en Argentina. Estos factores presentaron una alternativa de migración interna al propio Paraguay para los habitantes de Asunción y áreas cercanas, en lugar de dirigirse al Noreste o al Gran Buenos Aires (Bruno, 2008).

Esto último continuó y durante la década de 1980 la inmigración paraguaya mermó su ritmo histórico: el tamaño de la colectividad disminuyó 12.349 personas entre los censos de 1980 y 1991 (262.799 y 250.450, respectivamente) que señalaría no sólo una pausa en el flujo sino también un leve proceso de retorno. Como se dijo, ésto pudo obedecer a la significativa contracción en la economía Argentina y que Paraguay, al mismo tiempo, pudo mantener su producto bruto per cápita (Cerrutti, 2009). Sin embargo, y pese a esa reducción, la mayor cantidad de inmigrantes limítrofes aún provino desde Paraguay.

Asimismo, otra característica saliente fue que las mujeres se incorporaron aún más al fenómeno migratorio: en 1960 eran 101,8 varones por cada 100 mujeres, mientras que en 1980 esta relación descendió a 85,6.

En cuanto al período intercensal 1991-2001, coincidente con el Plan de Convertibilidad ${ }^{5}$, ocurrió un nuevo cambio en la tendencia: en esa década se produjo un fuerte incremento, equivalente a 2,6\% medio anual, en la llegada de paraguayos que se dirigieron directamente hacia grandes centros urbanos. Además, de acuerdo a los datos censales de 2001, se incrementó la feminización de la colectividad paraguaya (el índice de masculinidad descendió a 73,5).

Durante la década de 1990 el contexto fue marcado por la paridad cambiaria en Argentina y la mejora en su situación económica (evidenciada en el incremento del Producto Bruto per cápita). Este escenario, sumado al estancamiento en la economía

\footnotetext{
${ }^{4}$ La represa hidroeléctrica de Itaipú es un emprendimiento binacional entre Paraguay y Brasil que comenzó a construirse en enero de 1975 y el inicio de operaciones se produjo en Enero de 1984.

5 Regido por la Ley $\mathrm{N}^{\mathrm{o}} 23.928$, que establecía que un peso argentino equivalía a un dólar estadounidense.
} 
Torres. Paraguayos en Argentina: propensión a emigrar y características...

paraguaya, estimuló que se reanudara el flujo migratorio lo que se reflejó en el crecimiento de la comunidad paraguaya en Argentina: 325.046 en 2001, lo que equivale a un aumento del 24\% respecto de los contabilizados en 1991. Según explica Benencia (2000), los varones se insertaron en el sector de la construcción de obras de pequeña magnitud mientras que las mujeres lo hicieron en el servicio doméstico.

Cortés y Groisman (2004) mencionan que en el período 1990-1995 los migrantes limítrofes sustituyeron a los internos, pero en la etapa recesiva (cuando los sectores construcción y manufactura expulsaron mano de obra) ambos grupos sufrieron similares consecuencias. De este modo, los migrantes limítrofes reemplazaron a los internos en actividades como el servicio doméstico, la construcción y otras más de índole similar, ya que el empleo era de tipo no registrado, mientras que los migrantes internos tuvieron que comenzar actividades por cuenta propia. A diferencia de las décadas de $1970 \mathrm{y}$ 1980 (en las que la pobreza estructural estuvo más ligada a los migrantes limítrofes que a los internos), en el decenio de 1990 el desempleo y la pobreza aquejaron más a los migrantes internos.

Al respecto, los autores plantean que las características de los migrantes limítrofes e internos fueron comunes para las décadas de 1970 y 1980 (bajos niveles de instrucción y calificación). Sin embargo, en la década de 1990 los migrantes internos quedaron en inferioridad de condiciones con los limítrofes, ya que estos últimos mantuvieron las características que arrastraban de las dos décadas anteriores, mientras que los primeros sufrieron el deterioro de los niveles de escolarización. De este modo, los migrantes limítrofes fueron más demandados (especialmente en el sector de la construcción y de servicio doméstico) ya que ofrecían niveles de escolarización similar o a veces incluso superiores y con salarios y condiciones más flexibles que los nativos.

El fin de la Convertibilidad a principios de 2002 abrió una serie de interrogantes acerca si Argentina iba a continuar siendo un país atractivo para los migrantes paraguayos. El motivo de esto es que es de esperar que las fluctuaciones económicas en Argentina tengan fuertes efectos sobre la dinámica migratoria entre ambos países y la migración internacional se espera que responda rápidamente a variaciones en las condiciones macroeconómicas: períodos de recesión, alta inflación o deterioro de las condiciones de empleo en Argentina podrían reducir rápidamente el atractivo por parte de los migrantes internacionales (Parrado y Cerrutti, 2003).

Con la primera década del siglo XXI ya finalizada se encuentra evidencia que el tamaño de la población paraguaya en Argentina se incrementó nuevamente: los resultados del censo realizado en 2010 muestran que el tamaño de la población paraguaya alcanzó a 550.713 personas, un $69 \%$ mayor de lo que era en el 2001 y que implica un crecimiento medio anual de $6 \%$. Por su parte el índice de masculinidad creció por primera vez en muchos años: 79,7 hombres por cada 100 mujeres.

Otro aspecto interesante es el tamaño de la comunidad paraguaya es considerarla en relación a la población total de Argentina. Al respecto, Halpern (2008: 4) expresa que: 
La presencia de paraguayos en la Argentina aparece registrada, al menos en los datos oficiales, desde el primer censo nacional de 1869. En aquel momento, constituían el 0,2\% de la población total del país, el 1,6\% sobre la población extranjera y el 7,9\% sobre los inmigrantes de países limítrofes. Junto con estos últimos, conformaban el 2,6\% de la población total, porcentaje que se mantiene constante al día de hoy, a pesar de lo que muchos funcionarios, varios medios de comunicación y el imaginario general especulan [...] los paraguayos nunca llegaron a constituir siquiera el $1 \%$ de la población total. Al igual que en los censos de 1970 y de 1980, el de 2001 muestra que los paraguayos llegan al 0,9\%, marcando su momento de mayor proporción sobre el total del país.

Completando lo expuesto por el autor y actualizando con los datos censales de 2010, la proporción fue el 1,4\% del total país; alcanzando el máximo valor relativo. Asimismo, otros dos indicadores adquirieron su máximo valor histórico: el porcentaje de paraguayos respecto de la población extranjera total $(30,5 \%)$ y el porcentaje de paraguayos respecto de la población extranjera limítrofe $(44,2 \%)^{6}$.

En lo que respecto a la estructura etaria de los migrantes, en el Perfil Migratorio de Paraguay 2011 (ADEPO, 2011) se menciona que la proporción de 0 a 14 años es muy baja $(9,5 \%)$ y $11,8 \%$ corresponden a adultos mayores, siendo el grupo de edades activas (15 a 64 años) la más numerosa: 78,7\% $\%^{7}$. Asimismo, el Informe sostiene que en el 87,9\% de los casos la causa de la emigración se debió a motivos laborales ( $86 \%$ en el caso de los varones y casi $90 \%$ de las mujeres). Para aquellos con empleo en los lugares de origen, la categoría laboral que principalmente engloba a los varones es 'Empleados', mientras que en el caso de las mujeres es 'Trabajo doméstico'. En cuanto a la inserción laboral en el lugar de destino es porcentualmente mayor en los hombres que en las mujeres, quienes habitualmente lo hacen en el servicio doméstico bajo precarias condiciones de bajos salarios y falta de seguridad social.

En relación a la inserción laboral de los migrantes limítrofes en el área metropolitana de Buenos Aires, se realizó fundamentalmente entre los 20 y los 59 años de edad y se incorporaron en el sector servicios (37\%), actividades industriales y de construcción (29\%) y comercio (22\%). También rescata que el 33\% de los asalariados estaba en condiciones de precariedad laboral, medido a través de la falta de descuento para aportes de previsión social (Maguid, 1995).

En esa línea temática también se encuentra el trabajo de Cerrutti y Maguid (2007) donde, tomando como referencia los datos correspondientes a la Encuesta Permanente de Hogares del 2005, se probó que -aun siendo un año con recuperación económica-

\footnotetext{
${ }^{6}$ Los valores correspondientes a este censo y los demás están disponibles la Tabla 8 consignada el Anexo.

7 Como un diferencial de la corriente paraguaya que emigra a España, en este último caso los porcentajes son $14,7 \%, 5,9 \%$ y $79,4 \%$ para los grupos mencionados.
} 
Torres. Paraguayos en Argentina: propensión a emigrar y características...

los migrantes se insertaban en el mercado laboral a través de sectores económicos informales y precarios (v.g: construcción, servicio doméstico, calzado, industria textil, entre otros). A su vez, presentaron tasas de actividad más elevadas que los nativos dado que acceden a tareas mal remuneradas o no calificadas. Además, casi la mitad de las diferencias entre los ingresos promedio de los nativos y migrantes estaría explicada por los niveles educativos de los trabajadores y las formas de inserción laboral.

La marcada feminización del colectivo migratorio paraguayo constituye un importante motivo por el que se han realizado numerosos análisis alrededor de las mujeres. Bologna (2010) menciona que "una gran proporción de las mujeres se hallan ocupadas en el servicio doméstico, que es una actividad más estable; supone permanencia por períodos más prolongados y menos rotación entre empleadores que en la construcción" (Bologna, 2010: 207). Además, el autor sostiene que los varones habitualmente se ocupan en el sector de la construcción o algunas de sus actividades derivadas, pero debido a las redes de información existentes, es posible contratar a trabajadores desde el país de origen cuando resulta necesario.

Gaudio (2012) también estudió las mujeres paraguayas desde una perspectiva que vincula las decisiones y dinámica migratoria con los procesos familiares. En dicho trabajo, explora y describe cómo las inmigrantes paraguayas (ubicadas en Buenos Aires) experimentan la maternidad a distancia. A partir de las entrevistas realizadas surge como motivos de emigración las causas económicas, laborales o las vinculadas a situaciones familiares que impulsan la emigración.

Otro aspecto importante a tener en cuenta es el envío de remesas, estimándose que cerca de un tercio de las personas inmigrantes lo hace. Sin embargo, los jefes de hogar con experiencia migratoria vuelven a Paraguay con ahorros, con lo que el cálculo de remesas está subestimado debido a que no quedan registros de este flujo de dinero. $\mathrm{Al}$ analizar los factores que influyen positivamente en el envío de remesas de manera activa, aparecen la cantidad de viajes hacia Argentina y las intenciones de regresar. Por el contrario, el hecho de que sean documentados o no, como así también los años de residencia en Argentina, no modifica la probabilidad del envío de remesas. Al respecto, la muestra analizada no permite individualizar montos altos y/o considerables ni tampoco los medios utilizados para las remesas de dinero, esto es, giros bancarios, comisionistas, correos internacionales, amigos, colectiveros, y otros (Cerrutti y Parrado, 2007).

Por último, las regulaciones migratorias por parte del gobierno como así también las políticas económicas que modifican directamente el tipo de cambio alteran el valor relativo de las monedas locales y con ello el retorno inmediato esperado de la migración por parte de las personas, por ello los períodos de sobrevaluación del peso argentino pueden hacer de la migración internacional una inversión más atractiva (Parrado y Cerrutti, 2003).

Brevemente se puede señalar que durante 1998 se modificó la Ley de Migraciones vigente desde 1981 (la Ley General de Migraciones y Fomento de la Inmigración N ${ }^{o}$ 22.439, también conocida como "Ley Videla") mediante la firma del Convenio de Migración entre la República Argentina y la República del Paraguay. Sin embargo, 
el mismo fue rechazado por el Congreso paraguayo y -a pesar de poseer sanción del Senado argentino- no prosperó su aprobación en la Cámara de Diputados (Courtis, 2006; Novick, 2008). Posteriormente, en 2005, se implementó el Programa Nacional de Normalización Documentaria Migratoria. El fin principal del mismo es regularizar tanto la situación migratoria como así también la inserción e integración de los extranjeros residentes en forma irregular el país provenientes de Bolivia, Brasil, Chile, Colombia, Ecuador, Paraguay, Perú, Uruguay y República Bolivariana de Venezuela.

Al respecto, Pacecca y Courtis (2008: 46-47) expresan que:

la disposición prevé, para quienes acrediten un vínculo de parentesco con un argentino nativo o por opción o con un residente permanente, el otorgamiento de la residencia permanente; para el resto, la temporaria por dos años, convertible a permanente. El 'Patria Grande' también flexibiliza, en alguna medida, los requisitos para iniciar el trámite migratorio. Una forma de facilitar la presentación de la documentación es a través de un plan dos etapas: una fase inicial de acreditación de identidad (por medios amplios) y declaración jurada, a partir de la cual se obtiene, de manera gratuita, un certificado de residencia precaria con vigencia hasta la resolución del trámite, que constata el inicio del trámite migratorio y habilita a trabajar, estudiar, entrar, permanecer y salir del país; y una segunda etapa que conlleva, para completar la solicitud de la residencia temporaria, la certificación de carencia de antecedentes penales varios, el pago de la tasa migratoria ordinaria y la declaración de posesión de medios suficientes de subsistencia. Una característica del programa es su ejecución parcialmente descentralizada a través de convenios y/o acuerdos con los gobiernos provinciales y municipales y con instituciones de la sociedad civil, 'instituciones sociales colaboradoras' habilitadas para la recepción de trámites en sus propios establecimientos.

Según datos oficiales de la Dirección Nacional de Migraciones (Ministerio del Interior) para el mes de Agosto de 2010 se habían inscripto en el Programa 423.697 personas (283.789 en 2006, 120.687 en 2007 y 19.228 en 2008), se habían otorgado 98.539 radicaciones permanentes y 126.385 temporarias. En este ámbito, los inmigrantes paraguayos ocupan el mayor porcentaje en los tres rubros: el 58,6\% del total de inscripciones, el $47,92 \%$ de las radicaciones permanentes y el $60,6 \%$ de las radicaciones temporarias (Dirección Nacional de Migraciones, 2010).

Estos valores dan cuenta que aún queda la gran mayoría de los inmigrantes por tramitar la residencia. Esto podría explicarse de acuerdo a la opinión de Benencia 
Torres. Paraguayos en Argentina: propensión a emigrar y características...

(2009) quien señala que la resistencia a documentarse y radicarse en el país receptor se debe a un comportamiento asociado a conservar la pertenencia a su lugar de origen. En opinión del autor, en la mayoría de los casos la radicación resulta violenta ya que implica una renuncia (desde el punto de vista simbólico) a las raíces del inmigrante.

\subsection{Paraguayos en Argentina. Período 2001 - 2010}

Como se mencionó previamente, los últimos datos censales indican que en la primera década del siglo XXI no sólo no se detuvo el flujo migratorio paraguayo si no que incluso su tasa de crecimiento (6,03\% promedio anual) fue la mayor desde que se realizan los censos nacionales. De este modo, el tamaño de la comunidad paraguaya creció de 325.046 personas en 2001 a 550.713 en el 2010.

Analizando lo ocurrido en la situación económica de ambos países (mediante la diferencia en el producto bruto per cápita de Argentina y Paraguay) puede observarse que en el último decenio se registra una tendencia creciente en la discrepancia lo cual produce que la línea representada en el Gráfico 1 sea creciente en el último decenio, incluso superando la diferencia registrada en el segundo quinquenio de la década de 1990. Desde ya, este contexto no puede dejarse de lado a la hora de interpretar el crecimiento en el desplazamiento ocurrido en la última década en el que las oportunidades laborales representan un factor de atracción clave al momento de tomar la decisión de emigrar.

\section{Gráfico 1. Diferencia Producto Bruto per capita en dólares.} Argentina y Paraguay. 1965-2012

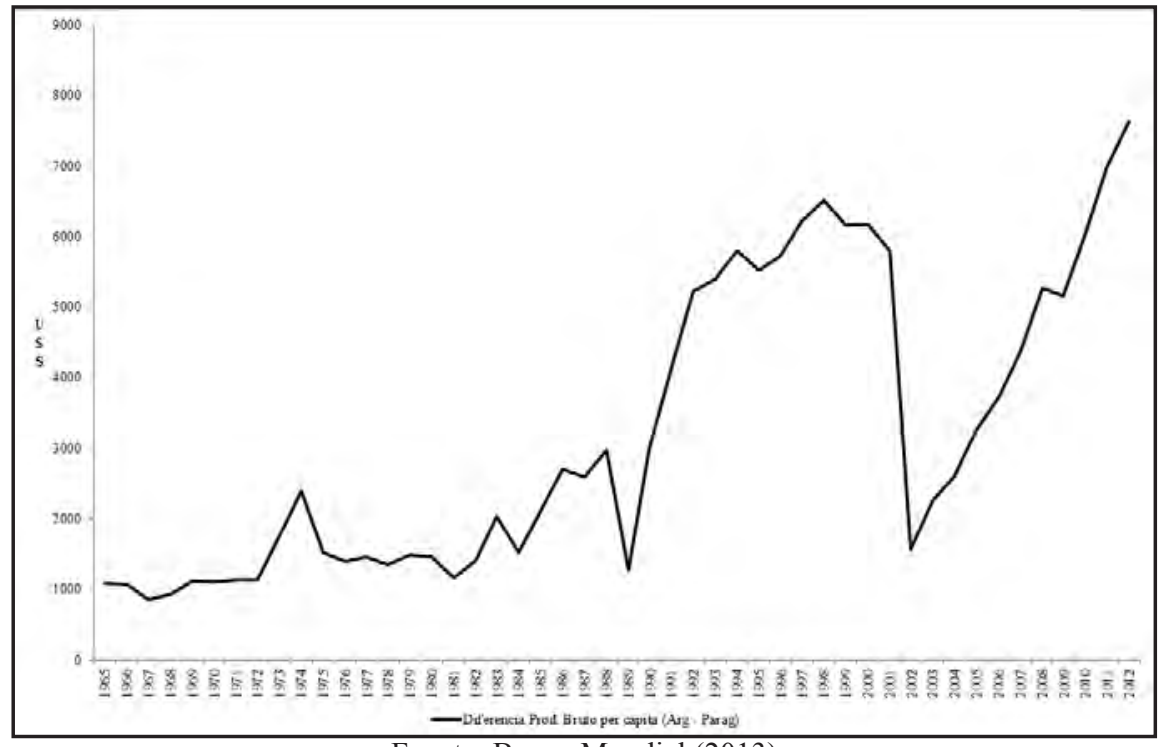

Fuente: Banco Mundial (2013)

Sin embargo, además hay un conjunto de características que cabe analizar más profundamente. Una de ellas es la vinculada a la estructura etaria, punto de partida para 
conocer si ha habido algún cambio en la composición por sexo y edad de la población. Asimismo, se ahonda en los sus lugares de destino seleccionados, lo relativo al nivel de instrucción y finalmente a aspectos de inserción laboral.

\subsubsection{Estructura poblacional}

Según surge del análisis de las pirámides (Gráfico 2), las edades centrales y la población femenina concentran el mayor porcentaje de la población. Por otra parte, en la pirámide del 2010 (a la derecha) se observa mayor participación de niños que respecto a la estructura del 2001 y también se incrementó la relación hombres/mujeres. La representación gráfica muestra que la base de la pirámide es estrecha debido a la baja participación relativa que tienen los niños en el total de los inmigrantes, presencia que se explica por acompañar a sus padres en el desplazamiento. Asimismo, es delgada la cúspide por lo que la población no es envejecida y es en las edades centrales (las laborales) donde se encuentra la mayor proporción poblacional.

Gráfico 2. Pirámide poblacional de nacidos en Paraguay. Argentina. 2001 y 2010

Paraguayos en Argentina: 2001

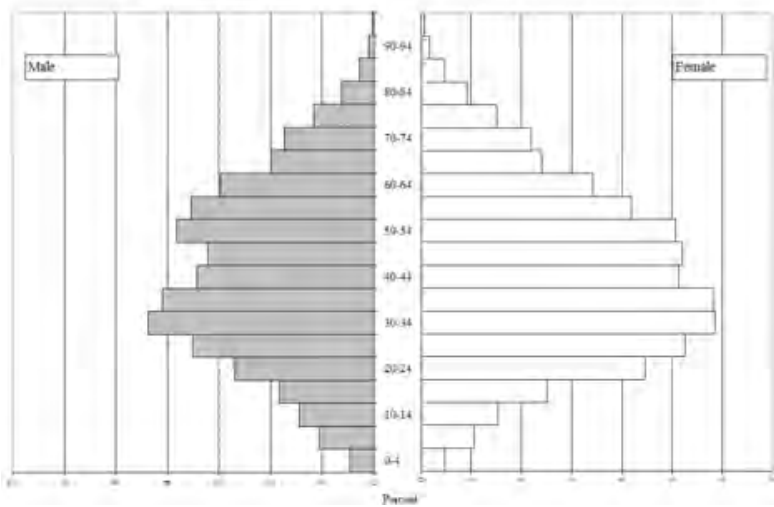

Paraguayos en Argentina: 2010

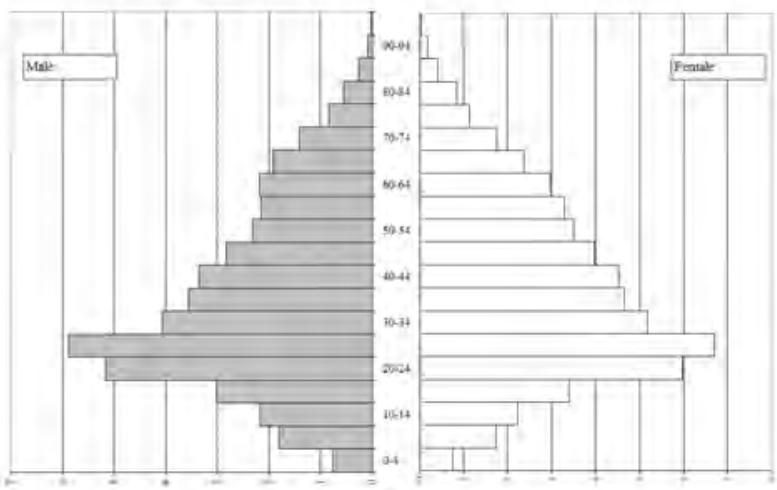

Fuente: INDEC. Censo Nacional de Población, Hogares y Viviendas (2001, 2010) 
Torres. Paraguayos en Argentina: propensión a emigrar y características...

La variación intercensal en la estructura etaria queda aún más expuesta al separar la población en tres grandes grupos: de 0 a 14 años, de 15 a 64 años y mayores de 64. Según se consigna en la Tabla 1, hay evidencia de mayor participación del grupo de 0 a 14 años (la diferencia respecto al censo de 2001 es de $3,7 \%$ en hombres y $3,2 \%$ en mujeres). Esta ganancia está explicada por el decrecimiento intercensal en los dos grupos etarios restantes y que implica el ensanchamiento observado en la base de la pirámide.

Tabla 1. Paraguayos en Argentina según grandes grupos de edad y sexo. 2001 y 2010

\begin{tabular}{|c|c|c|c|c|c|c|}
\hline & \multicolumn{2}{|c|}{ Masculino } & \multicolumn{2}{c|}{ Femenino } & \multicolumn{2}{c|}{ Total } \\
\hline Grupo de edad & 2001 & 2010 & 2001 & 2010 & 2001 & 2010 \\
\hline 0 a 14 años & $7,0 \%$ & $10,7 \%$ & $5,3 \%$ & $8,5 \%$ & $6,0 \%$ & $9,5 \%$ \\
\hline 15 a 64 años & $79,1 \%$ & $77,9 \%$ & $81,3 \%$ & $79,4 \%$ & $80,4 \%$ & $78,7 \%$ \\
\hline 65 años y más & $13,9 \%$ & $11,5 \%$ & $13,4 \%$ & $12,1 \%$ & $13,6 \%$ & $11,8 \%$ \\
\hline
\end{tabular}

Fuente: Elaboración propia en base a INDEC $(2006,2012)$

Como se expresó, el índice de masculinidad de los inmigrantes se incrementó en el último período intercensal. Como muestra el Gráfico 3, esto ha sucedido en todas las edades quinquenales hasta 49 años (especialmente entre 20 y 29 años, un grupo etario muy numeroso), mientras que de 50 años en adelante el índice disminuyó (y de mayor manera entre los 50 y los 64 años de edad). De manera similar, en todos los grupos quinquenales de edad aún el índice de masculinidad es inferior a 100 (que equivale a decir que las mujeres son más numerosas que los hombres), excepto para el de 5 a 9 años que con 100,6 supera muy levemente el umbral de mayoría numérica.

Gráfico 3. Índice de masculinidad de nacidos en Paraguay. Argentina. 2001 y 2010

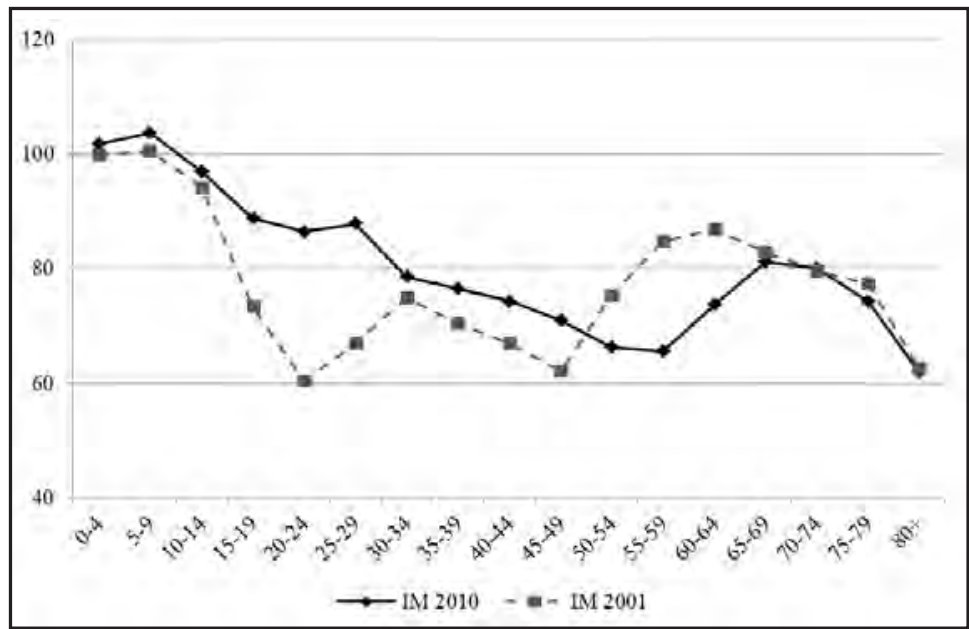

Fuente: Elaboración propia en base a INDEC $(2006,2012)$ 
La mayor cantidad de mujeres puede explicarse, en algunos casos, porque la decisión de emigrar fue tomada para mantener el vínculo familiar (acompañando al cónyuge) aunque en otras ocasiones lo hacen individualmente. Según los resultados censales, para las mujeres hay un decremento en la categoría 'Cónyuge o pareja' del $50,1 \%$ al $42,7 \%$ entre el censo 2001 y el efectuado durante 2010. Esta diferencia prácticamente se reparte en dos categorías que aumentan su valor: Hija (del 9,2\% al $13,5 \%$ ) y Jefe de Hogar (del 22,4\% al 26,5\%).

Asimismo, en el caso de los hombres entre ambos censos también se modificó la distribución de estas tres categorías: se percibe una importante disminución en 'Jefes de hogar' del 66,8\% al 52,9\% mientras que aumentó 'Hijo' (de 13,3\% a 18,6\%) y 'Cónyuge o pareja' de 6,3\% a 11,5\%. Esto estaría indicando un papel más activo de las mujeres en la faceta económica del hogar aunque esta hipótesis debería ser estudiada con mayor profundidad utilizando más información, como la relacionada al estado conyugal y si vive en pareja o matrimonio ${ }^{8}$.

\subsubsection{Distribución espacial de los inmigrantes paraguayos}

En materia de ubicación geográfica, en la Tabla 2 se percibe que entre el censo 2001 y el 2010 se produjo una concentración aún mayor a la ya existente en la zona del AMBA (lo que se explica por el decremento producido principalmente en Formosa y en Misiones) y en donde se encuentra prácticamente el $86 \%$ de los paraguayos. El aumento en el índice de masculinidad de 73,5 en el 2001 a 79,7 en 2010, además fue acompañado por el incremento de dicha relación en el AMBA (en Corrientes también, aunque su peso relativo es muy bajo) mientras que disminuyó en Formosa y Misiones.

Esto significa que en el último decenio intercensal la conducta de localización de los inmigrantes refleja el mismo patrón pero con una mayor concentración, probablemente debido al papel que juegan en el modo de contratación de mano de obra las redes sociales. Así, los que viven en el lugar de origen se relacionan con sus compatriotas conocidos ya asentados en el lugar de destino, y a partir de ahí comienza a funcionar la red colaborativa no sólo en lo ateniente a oportunidades laborales si no también de ayuda en la etapa de asentamiento en el nuevo lugar.

${ }_{8}$ Estas variables corresponden a la boleta del cuestionario ampliado del Censo 2010, cuyos resultados aún no han sido publicados. 
Torres. Paraguayos en Argentina: propensión a emigrar y características...

Tabla 2. Zonas de residencia e índice de masculinidad de paraguayos en Argentina. 2001 y 2010

\begin{tabular}{|c|c|c|c|c|c|c|c|c|}
\hline & \multicolumn{2}{|c|}{} & \multicolumn{2}{|c|}{} & \multicolumn{2}{c|}{$\begin{array}{c}\text { Índice de } \\
\text { Masculinidad }\end{array}$} \\
\cline { 2 - 10 } & 2001 & 2010 & 2001 & 2010 & 2001 & 2010 & 2001 & 2010 \\
\hline AMBA & 78,8 & 85,2 & 81,6 & 86,4 & 80,4 & 85,9 & 71 & 78,6 \\
\hline Corrientes & 0,9 & 0,6 & 0,9 & 0,6 & 0,9 & 0,6 & 68,6 & 80,2 \\
\hline Formosa & 6,9 & 3,7 & 6,1 & 3,7 & 6,4 & 3,7 & 83,6 & 80,5 \\
\hline Misiones & 8,6 & 5,1 & 6,9 & 4,6 & 7,6 & 4,9 & 92 & 88,3 \\
\hline Resto del país & 4,8 & 5,3 & 4,5 & 4,6 & 4,6 & 4,9 & 78,9 & 91,4 \\
\hline Total país & 100 & 100 & 100 & 100 & 100 & 100 & 73,5 & 79,7 \\
\hline
\end{tabular}

Fuente: Elaboración propia en base a INDEC (2006, 2012)

\subsubsection{Nivel de instrucción}

Este es otro elemento importante de incluir en el análisis ya que permite bosquejar sobre la posible inserción de los inmigrantes en el mercado laboral del lugar de destino, en particular sobre el sector económico y en las condiciones de empleo. En ese sentido, una primera aproximación muestra que es superior el porcentaje en el 2010, en relación al 2001, que sabe leer y escribir. Los resultados para cada censo fueron: $96 \%$ y 94,5 en el caso de los hombres y $96,1 \%$ y $94,2 \%$ en el de las mujeres (2010 y 2001, respectivamente).

Sala (2008) estudió el perfil educativo y laboral de los inmigrantes regionales arribados a Brasil y Argentina mediante el análisis de las personas de 20 y más años de edad que no asistían a establecimientos escolares al momento del censo 2001. Para conocer la situación existente en Argentina categorizó el nivel de instrucción en cuatro categorías: Muy bajo (hasta primaria incompleta), Bajo (primaria completa o secundaria incompleta), Medio (secundaria completa o terciaria o universitaria incompleta) o Alto (educación terciaria o universitaria completa).

Los resultados presentados en la Tabla 3 exponen que en ese año fue muy amplio el porcentaje de paraguayos con nivel educativo muy bajo o bajo $(87,3 \%$ y $85,7 \%$, hombres y mujeres, respectivamente). Al aplicar esas definiciones al censo 2010 , puede verse en la tabla que descendió mucho el porcentaje de hombres y mujeres con nivel de instrucción 'Muy Bajo'. Por contrapartida, creció el porcentaje del nivel 'Bajo' (sobre todo en mujeres) como así también el 'Nivel Medio', donde el incremento ha sido ostensiblemente mayor. Esto indicaría que los inmigrantes paraguayos han elevado su nivel de instrucción en esta última categoría donde antes no era tan importante: sólo alrededor de 1 cada 10 lograba llegar al nivel medio y en la actualidad el porcentaje se encuentra alrededor del doble de ese valor. 
Tabla 3. Nacidos en Paraguay, de 20 y más años de edad que no asistían a establecimientos educativos en la fecha de los censos, según sexo y máximo nivel de instrucción alcanzado. 2001 y 2010

\begin{tabular}{|c|c|c|c|c|}
\hline \multirow{2}{*}{ Nivel de Instrucción } & \multicolumn{2}{|c|}{ Hombres } & \multicolumn{2}{c|}{ Mujeres } \\
\cline { 2 - 5 } & 2001 & 2010 & 2001 & 2010 \\
\hline Muy bajo & $34,1 \%$ & $21,2 \%$ & $32,3 \%$ & $21,3 \%$ \\
\hline Bajo & $53,2 \%$ & $55,8 \%$ & $53,4 \%$ & $58,3 \%$ \\
\hline Medio & $10,8 \%$ & $20,9 \%$ & $11,8 \%$ & $22,2 \%$ \\
\hline Alto & $1,9 \%$ & $2,1 \%$ & $2,5 \%$ & $3,3 \%$ \\
\hline Total & $100 \%$ & $100 \%$ & $100 \%$ & $100 \%$ \\
\hline Total absoluto & 118,376 & 183,536 & 162,787 & 235,623 \\
\hline
\end{tabular}

Fuente: Elaboración propia en base a INDEC (2012) y Sala (2008)

\subsubsection{Tasas de actividad}

Según este indicador consignado en la Tabla 5, se observa que en el período comprendido entre 2001 y 2010 los migrantes paraguayos -hombres y mujeres- han incrementado su participación en el mercado laboral. Esto puede explicarse por el mayor peso relativo que en el 2010 presenta el grupo de edad de 20 a 29 años, que es fuertemente activo en términos laborales.

Además, los valores expuestos en la Tabla 4 señalan que las tasas de actividad de los inmigrantes son superiores a la de los nativos argentinos en ambos censos, pero la diferencia se incrementó mayormente en los hombres en 2010.

Sin embargo, aún resta por ahondar otros aspectos relativos al último censo, como ser la rama de actividad, categoría ocupacional, calificación de la tarea, sector ocupacional, cobertura de salud, descuentos jubilatorios, datos que todavía no están disponibles y que permitirían tratar más profundamente este aspecto.

Tabla 4. Población de 14 y más años de edad. Tasas de actividad, según nativos y paraguayos. 2001 y 2010

\begin{tabular}{|c|c|c|c|c|}
\hline & \multicolumn{2}{|c|}{2001} & \multicolumn{2}{c|}{2010} \\
\hline & Nativos & Paraguayos & Nativos & Paraguayos \\
\hline Hombres & $70,6 \%$ & $74,6 \%$ & $77,4 \%$ & $84,9 \%$ \\
\hline Mujeres & $45,1 \%$ & $52,5 \%$ & $54,6 \%$ & $62,3 \%$ \\
\hline Total & $57,4 \%$ & $61,7 \%$ & $65,5 \%$ & $72,2 \%$ \\
\hline
\end{tabular}

Fuente: Elaboración propia en base a INDEC (2006 y 2012)

\section{Materiales y métodos}

En el presente trabajo se utiliza una fuente de datos primaria proveniente de encuestas de hogares relevadas en Paraguay durante el año 2008 a residentes de cuatro 
Torres. Paraguayos en Argentina: propensión a emigrar y características...

departamentos: Central, Itapúa, Caaguazú y Alto Paraná. En total, se encuestaron 546 hogares y se obtuvo información de 2.591 personas.

El trabajo realizado es producto de un estudio interdisciplinario integrado por la Asociación Paraguaya de Estudios de Población (ADEPO), el Centro de Estudios Avanzados (CEA/CONICET, Universidad Nacional de Córdoba, Argentina), el Instituto de Investigaciones Geohistóricas (IIGHI CONICET, Argentina) y la Agencia Nacional de Promoción Científica y Técnológica de Argentina. Además se contó con el apoyo de Dirección General de Encuestas, Estadísticas y Censos de Paraguay (DGEEC) y el Fondo de Población de las Naciones Unidas (UNFPA).

En relación a los aspectos metodológicos implementados en el relevamiento, la documentación provista por $\mathrm{ADEPO}$ señala que:

La investigación corresponde a una de corte transversal cuya población de estudio comprende a los hogares con alguna experiencia migratoria. Por tanto el diseño muestral se enmarca dentro de un método cuasi-aleatorio, por el hecho de que la primera etapa consta de una selección no aleatoria de departamentos seleccionados, conforme a la concentración de hogares receptores de remesas; la segunda etapa de selección corresponde a distritos y localidades que en 50\% han sido tomadas de la EPH 2007 (distritos donde estén localizados hogares que han recibido remesas, lo cual remite a hogares que han tenido miembros que han emigrado) y el 50\% restante han sido seleccionados al azar. En la tercera etapa se han elegido los hogares de forma aleatoria. Se ha tratado de asegurar el carácter aleatorio de la muestra a través de la ubicación al azar de los hogares y el $50 \%$ de los distritos que no han sido incorporados en la EPH 2007 (ADEPO, 2011:10).

De acuerdo al estudio, en una primera etapa se tomaron los departamentos de Central, Caaguazú e Itapúa donde se concentra el $53 \%$ de los hogares que reciben remesas resultando seleccionados 400 hogares. Finalmente, para lograr la mejor representación posible de la experiencia migratoria, se seleccionaron 100 hogares del departamento Alto Paraná, que es el cuarto en orden de recepción de remesas. De acuerdo a esto, la distribución final de la muestra de hogares tomada según departamentos se presenta en la Tabla 5. 
Tabla 5. Distribución muestral por áreas de residencia. Paraguay. 2008

\begin{tabular}{|c|c|c|c|}
\hline Departamento & Total & Urbana & Rural \\
\hline Caaguazú & 108 & 52 & 56 \\
\hline Itapúa & 120 & 60 & 60 \\
\hline Central & 210 & 152 & 58 \\
\hline Alto Paraná & 108 & 63 & 45 \\
\hline Total & 546 & 327 & 219 \\
\hline \multicolumn{4}{|c}{ Fuente: ADEPO (2009) }
\end{tabular}

El propósito del proyecto fue recabar información de las características de las viviendas y de los miembros del hogar con alguna experiencia migratoria. En este marco, resultó de interés indagar sobre un conjunto de aspectos particulares de la migración: los factores motivacionales (cambios en los contextos políticos y sociales del Paraguay en relación a los países de destino), el rol que juegan las migraciones en las estrategias de diversificación de las actividades económicas de los hogares, la transmisión intergeneracional de la cultura migratoria y finalmente, el estudio del posible impacto remesas respecto del desarrollo del país y su contribución en la lucha contra la pobreza.

En el cuestionario se preguntó (además de las características del hogar y la vivienda) sobre migración interna y acerca de conocidos residentes en otros países. Asimismo se consultó sobre la experiencia migratoria de todos los miembros del hogar al exterior y un detallado cuestionario sobre la primera y la última experiencia migratoria del jefe de hogar. Este bloque aborda aspectos relacionados con su situación al momento de su salida: edad, estado civil, si tenía hijos, situación laboral, con quién viajó, si tenía conocidos en el exterior y si obtuvo ayuda de ellos, y el modo en que accedió a su primer trabajo, entre otros temas.

Yendo al trabajo realizado en el presente artículo, la metodología empleada consiste en estimar un modelo de regresión logística binaria donde la variable dependiente apunta a si el jefe de hogar emigró para vivir o trabajar en Argentina, estableciéndose el valor de dicha variable del siguiente modo:

0 si el jefe del hogar nunca emigró hacia Argentina para vivir y/o trabajar.

1 si en algún período de su vida el jefe del hogar emigró hacia Argentina para vivir $\mathrm{y} / \mathrm{o}$ trabajar.

Por su parte, las variables independientes incluidas en el modelo capturaron la situación de cada una de ellas al momento en que el jefe de hogar emigró, para aquellos que lo hicieron, y en el caso que nunca lo hayan hecho se utilizó la información al momento en que se realizó la encuesta sobre9: 1) Sexo (Femenino/Masculino) 2) Edad (Menor de 30 años/30 a 44 años/45 a 59 años/60 años o más) 3) Lugar de residencia (Zona rural/Zona urbana) 4) Cantidad de hijos: (Sin hijos/Con hijos) 5) Situación laboral (No trabajaba/Trabajaba) 6) Estado conyugal (Unido o casado/Otra situación).

\footnotetext{
9 En todos los casos, la última categoría de cada variable es la utilizada como referencia en la regresión.
} 
Torres. Paraguayos en Argentina: propensión a emigrar y características...

En consecuencia, a partir del valor que brindan los coeficientes de la correspondiente ecuación de regresión surge la interpretación del efecto que cada una de las variables (o factores) desempeña en la propensión a emigrar de cada jefe de hogar.

\section{Propensión a emigrar: discusión y resultados}

Ya abordado uno de los objetivos principales de este trabajo (presentar las principales características sociodemográficas de la comunidad paraguaya según los resultados del censo 2010), puede avanzarse con el segundo de ellos: examinar la dinámica migratoria teniendo en cuenta el contexto de los jefes de hogar paraguayos (situación laboral y conyugal, tener hijos y edad, entre otras).

Sin embargo, el trabajo realizado por Cerrutti y Parrado (2006) constituye un valioso antecedente al presente, ya que en él se describe la dinámica de la migración paraguaya a la Argentina con especial énfasis en los contextos socioeconómicos que la determinan, estimando para ello: "I) modelos de probabilidad (modelos de evento discreto) de primera migración antes del casamiento para varones y mujeres; II) de probabilidad de emigrar (y su forma) de los miembros de la pareja luego del matrimonio; y por último III) de probabilidad de distintas formas de residencia de la pareja tanto en Argentina como en Paraguay" (Cerrutti y Parrado, 2006: 108).

Allí, los resultados muestran que a lo largo de la vida las probabilidades migratorias de los hombres y mujeres -jefes de hogar en ambos casos- en los distritos de Carapeguá y San Roque González son elevadas, aunque las mujeres emigran en menor proporción que los hombres ${ }^{10}$.

En segundo lugar, las probabilidades de emigración son mucho más elevadas en Carapeguá que en San Roque González, tanto en mujeres como en hombres de edades tempranas antes del casamiento. En el caso de los hombres carapagüenses, la actividad laboral también influiría ya que si éstos trabajan en la construcción o como macateros las probabilidades de emigrar antes de casarse son inferiores que si no tienen trabajo; y por el contrario, son considerablemente mayores en caso que trabajen en la industria del cuero o calzado. Asimismo, en estas edades tempranas, los factores vinculados con las redes sociales (como la presencia de hermanos en Argentina) pesan más en la probabilidad de emigrar que el nivel educativo o su condición de residencia urbana o rural; mientras tanto el poseer una vivienda propia influye negativamente.

Por su parte, en el caso de las mujeres el bajo número de eventos migratorios torna los resultados poco confiables pero en algunos casos se dirigen en línea con el de los varones. Por ejemplo, las mujeres productoras de artesanías y actividades manuales calificadas o semicalificadas presentan una probabilidad de emigrar muy inferior antes de casarse mientras que contar con una vivienda la disminuye. Pero en contraste con los hombres, poseer mayor nivel de instrucción influye negativamente, así como la residencia rural parece estimular la migración femenina más que la masculina.

\footnotetext{
${ }^{10}$ En opinión de los autores, esto puede deberse a que debido a la metodología implementada las mujeres migrantes estés subrepresentadas.
} 
Finalmente, las mujeres de ambos distritos tienen la misma probabilidad de emigrar antes y después de casarse pero contar con hermanos en Argentina provoca un efecto más débil que en los hombres en lo referido a la migración antes del primer casamiento.

En cuanto al contexto de las parejas ya constituidas, los factores asociados a las decisiones migratorias, tanto de varones como de mujeres, se vinculan fuertemente con la experiencia migratoria previa. Además, se resalta que a medida que transcurren los años a partir de la unión las probabilidades de la primera migración disminuyen; al igual que el número de niños constituye otro limitante. Por último, las parejas que pertenecen a áreas rurales cuentan con mayor probabilidad de que su primera migración sea realizada por ambos integrantes.

Por otro lado, y previo a revisar los resultados, es prudente mencionar una aclaración: en base al módulo de historia migratoria contenido en la encuesta se dispone de datos referidos a jefes de hogar que nunca emigraron o que lo hicieron pero retornaron a Paraguay, por lo que no se cuenta con información de aquellos que no regresaron cuando se llevó a cabo la encuesta. Esto implica que probablemente los emigrantes considerados en el análisis posean diferentes características de los que no retornaron. Desde ya, lo óptimo sería poder comparar los resultados entre ambos grupos para realizar un análisis más completo, aunque lamentablemente esto no es posible con esta fuente de datos. Por lo tanto, los resultados deben ser interpretados teniendo en cuenta esta consideración.

Aclarado lo anterior, los datos contenidos en la columna "Exp(B)" de la Tabla 7 permiten interpretar los resultados de la regresión logística que estima la emigración de jefes de hogar hacia Argentina. En base a los coeficientes obtenidos, se desprende que:

- Tener menos de 30 años de edad es la característica que mayor incidencia posee ya que el odds de emigrar es 19,20 veces más alto comparado con aquellos que tenían 60 años o más, mientras que el odds de los que tenían entre 30 y 44 años de edad fue 9,50 veces superior. Aquellos que tenían entre 45 y 59 años registraron un odds 3,86 veces más alto que aquellos que tenían 60 años o más, lo que permite concluir que, según este análisis estadístico, la edad de la persona resulta ser el factor que más incide a favor de que una persona emigre. Tal como se menciona en el informe elaborado por ADEPO (2008), si bien el promedio de edad al momento de la emigración es superior a los 30 años, el odds de emigrar es superior para aquellos más jóvenes.

- El no poseer hijos versus tener incrementa el odds de emigrar en 2,86 veces.

- No tener empleo incrementa el odds de emigrar hacia Argentina 4,14 veces con respecto a los que poseen.

- Aquellos que no estaban casados o unidos registraron un odds de emigrar 2,38 veces más alto respecto de los que lo estaban.

- En el caso del lugar de residencia, aquellos que vivían en zonas rurales experimentaron un odds de emigrar 1,32 veces mayor que los que lo hacían 
Torres. Paraguayos en Argentina: propensión a emigrar y características...

en zonas urbanas. Sin embargo, esta variable no presentó significancia estadística.

- $\quad$ El hecho de ser mujer disminuye el odds de emigrar a Argentina ya que el mismo es 0,65 veces el de los varones, aunque hay que tener en cuenta que esta variable tampoco resultó significativa en el modelo.

De este modo, según se observa en la síntesis efectuada en los párrafos previos, los resultados de la propensión emigratoria exhibidos en este trabajo son similares a los obtenidos por Cerrutti y Parrado, y ponen de relieve el modo en que en las edades tempranas la probabilidad de emigrar es superior. Asimismo, otros factores que forman parte del contexto también estimulan (o desalientan) la emigración: en particular lo que sucede con la unión familiar y el empleo.

En cuanto a la incidencia de la conformación del matrimonio, existe un diferencial en el patrón migratorio ya que la propensión a emigrar es superior luego de haberse casado o unido.

Pero, a diferencia del estudio presentado por Cerrutti y Parrado, ni las variables sexo ni lugar de residencia (medido como rural o urbano) resultaron estadísticamente significativas. Esto posiblemente esté explicado, aunque sea en parte, porque el módulo de historia migratoria fue relevado a los jefes de hogar que nunca salieron del país o a aquellos que retornaron. Queda como un interrogante si los resultados serían diferentes al incluir lo referido a aquellos que emigraron y no volvieron. Igualmente, hay otras variables que no pudieron ser incluidas en el análisis ya que no estaban; particularmente el nivel de instrucción y el sector laboral, de manera de conocer si hay sectores que propicien la búsqueda de nuevos horizontes.

\section{Consideraciones finales}

La migración paraguaya en Argentina es un antiguo proceso que cobró mayor notoriedad a partir de mediados del siglo pasado y en la actualidad se encuentra en un momento que no tiene precedentes acerca del tamaño de la comunidad. Como ya se señaló en estas páginas, se mencionan las principales características que tuvo a lo largo del tiempo y lo ocurrido en la última década con la intención de conocer el estado del proceso en la actualidad. Se destaca que en el último decenio se ha registrado una tendencia creciente en la diferencia entre los productos brutos per cápita de ambos países (incluso mayor de la experimentada en el último quinquenio de la década de 1990) lo cual representa un importante factor de atracción por las expectativas laborales que se derivan en este escenario.

También ha habido algunas modificaciones en la última década: se incrementó el índice de masculinidad en toda la comunidad y en cada una de las edades quinquenales, ganó más peso relativo el grupo de edades centrales comprendido entre 15 y 64 años de edad, se observa mayor nivel de instrucción tanto en hombres como mujeres, se evidencia mayor concentración geográfica en el área del AMBA y finalmente, también es superior la tasa de actividad de hombres y mujeres. 
Asimismo, según el resultado a través de la estimación del modelo de regresión logística binaria, se observa que la probabilidad de emigrar afecta de manera más fuerte a los jóvenes, aquellos que están desempleados, no tienen hijos y que aún no han constituido matrimonio. Sin embargo, no resultó significativo ser varón o mujer como así tampoco vivir en el área rural o urbana. Sin embargo, al no disponer de la información perteneciente a aquellos que emigraron pero aún no han vuelto, estos resultados podrían variar de contar con esos datos.

Finalmente, la dinámica migratoria paraguaya no sólo está explicada por una importante diferencia en los mercados de trabajo del AMBA y de las otras provincias si no también que se atribuye al. proceso social que a lo largo del tiempo ha construido una sólida red que se retroalimenta en la que se presta mutua ayuda a la hora de conseguir empleo y asentarse en el lugar de destino. Como prueba de ello, se ha producido una mayor concentración de los migrantes en el AMBA, menguando el desplazamiento hacia lugares que en el pasado tenían mayor importancia, como las provincias de Misiones y Formosa.

\section{Referencias bibliográficas}

Asociación Paraguaya de Estudios de Población (ADEPO), UNFPA. 2009. Características de la Reciente Emigración Paraguaya. Principales resultados de una encuesta sobre emigración (2008). Asunción, Paraguay.

Asociación Paraguaya de Estudios de Población (ADEPO). 2011. Perfil Migratorio de Paraguay. Buenos Aires, Organización Internacional para las Migraciones.

Argentina. Ley de Migraciones $N^{o}$ 25.871, Poder Legislativo Nacional. Reglamento Migratorio: Decreto 616/2010. Boletín Oficial 21/01/04.

Banco Mundial. 2013. "GDP per capita (current US\$)”, [En línea] URL: http://data. worldbank.org/indicator/NY.GDP.PCAP.CD acceso el 19 de octubre de 2013.

Benencia, Roberto. 2000. "Argentina: la problemática social de la migración limítrofe". En: Comercio exterior, Vol. 50, No 3, México D. F., Banco Nacional de Comercio Exterior, pp. 251-257.

Benencia, Roberto. 2007. "La migración limítrofe". En Torrado Susana (comp.) Una historia social del siglo XX. Tomo I, Buenos Aires, Edhasa, pp. 571-599.

Benencia, Roberto. 2009. “Apéndice: La inmigración limítrofe”. En: Devoto Fernando Historia de la inmigración en la Argentina, Buenos Aires, pp. 433-484.

Bologna, Eduardo. 2010. "Migraciones entre países del sur: Los cambios y las continuidades en los flujos limítrofes hacia Argentina". En: Migraciones Internacionales, Vol. 5, No 3, El Colegio de la Frontera Norte, México, pp. 175209.

Cacopardo, María C. 2000. “Mujeres migrantes y jefas de hogar”. En: Mujeres en Escena, Actas de las V Jornadas de Historia de las Mujeres y Estudios de Género, Santa Rosa, Universidad Nacional de la Pampa. 
Torres. Paraguayos en Argentina: propensión a emigrar y características...

Cacopardo, María C. 2002. "Mujeres migrantes y trabajadoras en distintos contextos regionales urbanos". En: Papeles de Población $\mathrm{N}^{\mathrm{o}}$ 34, Toluca, Universidad Autónoma del Estado de México, pp. 135-157.

Cacopardo, María C. 2004. "Crisis y mujeres migrantes en la Argentina", comunicación presentada en el II Seminario de la "Red de Estudios de Población", Centre d'Estudis Demografics, febrero.

Cacopardo, María C. y Maguid, Alicia. 2001. "Argentina: International Migrants and Gender Inequality in the Labor Market”. XXIV General Population Conference, IUSSP, San Salvador de Bahía [En línea] URL: http://www.archive-iussp.org/ Brazil2001/s20/S26_02_Maguid.pdf acceso el 22 de junio de 2013.

Carrón, Juan M. 1979. "Shifting Patterns in Migration from Bordering Countries to Argentina: 1914-1970". En International Migration Review, Vol. 13, № 3. The Center for Migration Studies of New York, New York, pp. 475-485.

Cerrutti, Marcela. 2009. "Diagnóstico de las poblaciones de inmigrantes en la Argentina". En: Serie de documentos de la Dirección Nacional de Población, Nº 2, Dirección Nacional de Población, Secretaría del Interior, Buenos Aires.

Cerrutti, Marcela y Alicia Maguid. 2007. "Inserción laboral e ingresos de migrantes limítrofes y del Perú en el Área Metropolitana de Buenos Aires, 2005”. En: Notas de Población, $N^{\circ}$ 83, Santiago de Chile, pp. 75-98.

Cerrutti, Marcela y Emilio Parrado. 2006. "Migración de Paraguay a la Argentina: género, trabajo y familia en contextos de origen diferenciados". En: Grimson, A. y Jelin, E. (comp.), Migraciones regionales hacia la Argentina. Diferencia, desigualdad y derechos, Buenos Aires, Prometeo Libros, pp. 99-133.

Cerrutti, Marcela y Emilio Parrado. 2007. "Remesas enviadas por inmigrantes paraguayos en Argentina: prevalencia, montos y usos”. En: Integración y Comercio, $\mathrm{N}^{\mathrm{o}} 27$, pp. 21-46.

Cortés, Rosalía y Fernando Groisman. 2004. "Migraciones, mercado de trabajo y pobreza en el Gran Buenos Aires". En: Revista de la CEPAL, No 82, Santiago de Chile, pp. 173-191.

Courtis, Corina. 2006. "Hacia la derogación de la Ley Videla: la migración como tema de labor parlamentaria en la Argentina de la década de 1990". En: Grimson, Alejandro y Jelin, Elizabeth (comps.) Migraciones regionales hacia la Argentina. Diferencia, desigualdad y derechos, Buenos Aires, Prometeo, pp. 169-205.

Dirección Nacional de Migraciones. 2010. "Informe estadístico del Programa Nacional de Normalización Documentaria Migratoria" [En línea], URL: http://www. migraciones.gov.ar/pdf_varios/estadisticas/Patria_Grande.pdf acceso 10 de septiembre de 2013.

Gaudio, Magalí. 2012. "Mujeres paraguayas en el Área Metropolitana de Buenos Aires. Decisión migratoria, relaciones familiares y maternidad a distancia". En: Revista Temas de Antropología y Migración, № 3, Universidad de Buenos Aires, Buenos Aires, pp. 40-60. 
Halpern, Gerardo. 2008. "Una aproximación a los paraguayos organizados en Buenos Aires", Sociedad, No 27 [En línea] Inmigrantes, URL: http://www.sociales. uba.ar/wp-content/uploads/12.-Una-aproximaci\%C3\%B3n-a-los-paraguayosorganizados-en-Buenos-Aires.pdf acceso el 01 de septiembre de 2013.

Hosmer, David y Stanley Lemeshow. 1989. Applied Logistic Regression. New York, John Wiley y Sons.

INDEC. 1993. Censo Nacional de Población y Viviendas 1991. Buenos Aires, Instituto Nacional de Estadísticas y Censos.

INDEC. 1996. La población no nativa de la Argentina, 1869-1991. En: Serie Análisis Demográfico $\mathrm{N}^{0}$ 6, Buenos Aires.

INDEC. 2006. Censo Nacional de Población, Hogares y Viviendas 2001. Buenos Aires, Instituto Nacional de Estadísticas y Censos.

INDEC. 2012. Censo Nacional de Población, Hogares y Viviendas 2010. Buenos Aires, Instituto Nacional de Estadísticas y Censos.

Maeder, Ernesto J. A. 2010. "La frontera argentino-paraguaya. Etapas de su delimitación. (1618-1950)". En: Folia Histórica del Nordeste, No 18. Resistencia, Instituto de Investigaciones Geohistóricas, Conicet e Instituto de Historia, UNNE, pp. 7-32.

Maguid, Alicia. 1990. Migrantes limítrofes en la Argentina: perfil-socioeconómico y ocupacional en 1980. Total del país. Buenos Aires, Ministerio del Interior- Fondo de las Naciones Unidas para actividades de población.

Maguid, Alicia. 1995. "Migrantes limítrofes en la Argentina: su inserción e impacto en el mercado de trabajo". En: Estudios del Trabajo, No 10, Buenos Aires, Aset, pp. 47-76.

Maguid, Alicia. 1997. "Migrantes limítrofes en el mercado de trabajo del Área Metropolitana de Buenos Aires. 1980-1996". En: Estudios Migratorios Latinoamericanos. Año 12, N 35, Buenos Aires, Centro de Estudios Migratorios Latinoamericanos (CEMLA), pp. 31-62.

Maletta, Héctor. 1992. "Migración internacional en Paraguay e integración del Cono Sur: una agenda de investigación”. En: Revista de la OIM sobre Migraciones en América Latina, Vol. 10, $\mathrm{N}^{\mathrm{0}} 10, \mathrm{~N}^{\mathrm{o}} 2 / 3$, pp. 5-51.

Marshall, Adriana. 1983. "Las migraciones de países limítrofes en la Argentina". En: Memorias del Congreso Latinoamericano de Población y Desarrollo, México, El Colegio de México, UNAM, PISPAL.

Marshall, Adriana y Orlansky, Dora. 1981. "Las condiciones de expulsión en la determinación del proceso emigratorio desde países limítrofes hacia la Argentina". En: Desarrollo Económico, Vol. 20, № 30, pp. 491-510.

Meichtry, Norma y Beck, Hugo. 2002. “Caracterización demográfica de la inmigración limítrofe en el nordeste de Argentina”. En: Silva, H. A. Historia de las Migraciones Limitrofes en el Cono Sur de América. Argentina, Bolivia, Brasil, Chile, Paraguay y Uruguay, Primera Parte - Del Siglo XIX a 1960. Tomo I - Argentina. México, Instituto Panamericano de Geografía e Historia, pp. 145-183. 
Torres. Paraguayos en Argentina: propensión a emigrar y características...

Novick, Susana. 2008. Migración y políticas en Argentina: Tres leyes para un país extenso (1876-2004). URL: http://webiigg.sociales.uba.ar/pobmigra/archivos/ Novick8-8-08.pdf acceso el 18 de agosto de 2013.

Pacecca, María Inés y Courtis, Cristina. 2008. "Inmigración contemporánea en Argentina: dinámicas y políticas". En: Serie Población y Desarrollo $N^{0} 84$, Santiago de Chile, CEPAL.

Parrado, Emilio y Cerrutti, Marcela. 2003. "Labor Migration between Developing Countries: The Case of Paraguay and Argentina". En: International Migration Review, Vol. 37, No 1 (Spring, 2003), The Center for Migration Studies of New York, New York, pp. 101-132.

Sala, Gabriela. 2008. "Perfil educativo y laboral de los nuevos y viejos migrantes regionales censados en Argentina y Brasil". En: Migraciones Internacionales Vol. 4, $\mathrm{N}^{\circ}$ 4, julio-diciembre, México, pp. 73-106. 


\section{ANEXO}

Tabla 6. Argentina: Evolución de la distribución porcentual de residentes paraguayos, 1914-2010

\begin{tabular}{|c|c|c|c|c|c|c|c|c|}
\hline Lugar de residencia & 1914 & 1947 & 1960 & 1970 & 1980 & 1991 & 2001 & 2010 \\
\hline Buenos Aires & 11,2 & 13,3 & 29,6 & 58,4 & 65,6 & 70,8 & 80,4 & 85,9 \\
\hline Formosa y Misiones & 46,2 & 62,4 & 54,8 & 31,3 & 26,1 & 21,8 & 14,1 & 8,5 \\
\hline Otras Provincias & 42,6 & 24,3 & 15,6 & 10,2 & 8,3 & 7,4 & 5,5 & 5,6 \\
\hline
\end{tabular}

Fuente: Carrón (1979) y elaboración propia en base a INDEC

Tabla 7. Estimación del modelo de regresión logística que predice la probabilidad que un jefe de hogar paraguayo emigre a Argentina. 2008

\begin{tabular}{|c|c|c|c|c|c|c|}
\hline & $B$ & E.T. & Wald & DF & Sign. & Exp(B) \\
\hline Sit. Cony. (No Casado/Unido) & 0,869 & 0,33 & 7,139 & 1 & 0,008 & 2,384 \\
\hline Edad & & & 42,793 & 3 & 0,000 & \\
\hline Edad(<30) & 2,992 & 0,51 & 34,003 & 1 & 0,000 & 19,920 \\
\hline Edad(30-44) & 2,251 & 0,50 & 20,328 & 1 & 0,000 & 9,495 \\
\hline Edad(45-59) & 1,350 & 0,52 & 6,841 & 1 & 0,009 & 3,856 \\
\hline Cantidad De Hijos (Sin Hijos) & 1,050 & 0,36 & 8,584 & 1 & 0,003 & 2,859 \\
\hline Sit. Laboral (No Trabajaba) & 1,420 & 0,30 & 22,244 & 1 & 0,000 & 4,138 \\
\hline Área (Rural) & 0,280 & 0,26 & 1,152 & 1 & 0,283 & 1,324 \\
\hline Sexo (Mujer) & $-0,470$ & 0,35 & 1,800 & 1 & 0,180 & 0,625 \\
\hline Constante & $-4,196$ & 0,51 & 67,080 & 1 & 0,000 & 0,015 \\
\hline
\end{tabular}

Fuente: Elaboración propia en base a la Encuesta sobre Migración en Paraguay (2008) 
Torres. Paraguayos en Argentina: propensión a emigrar y características...

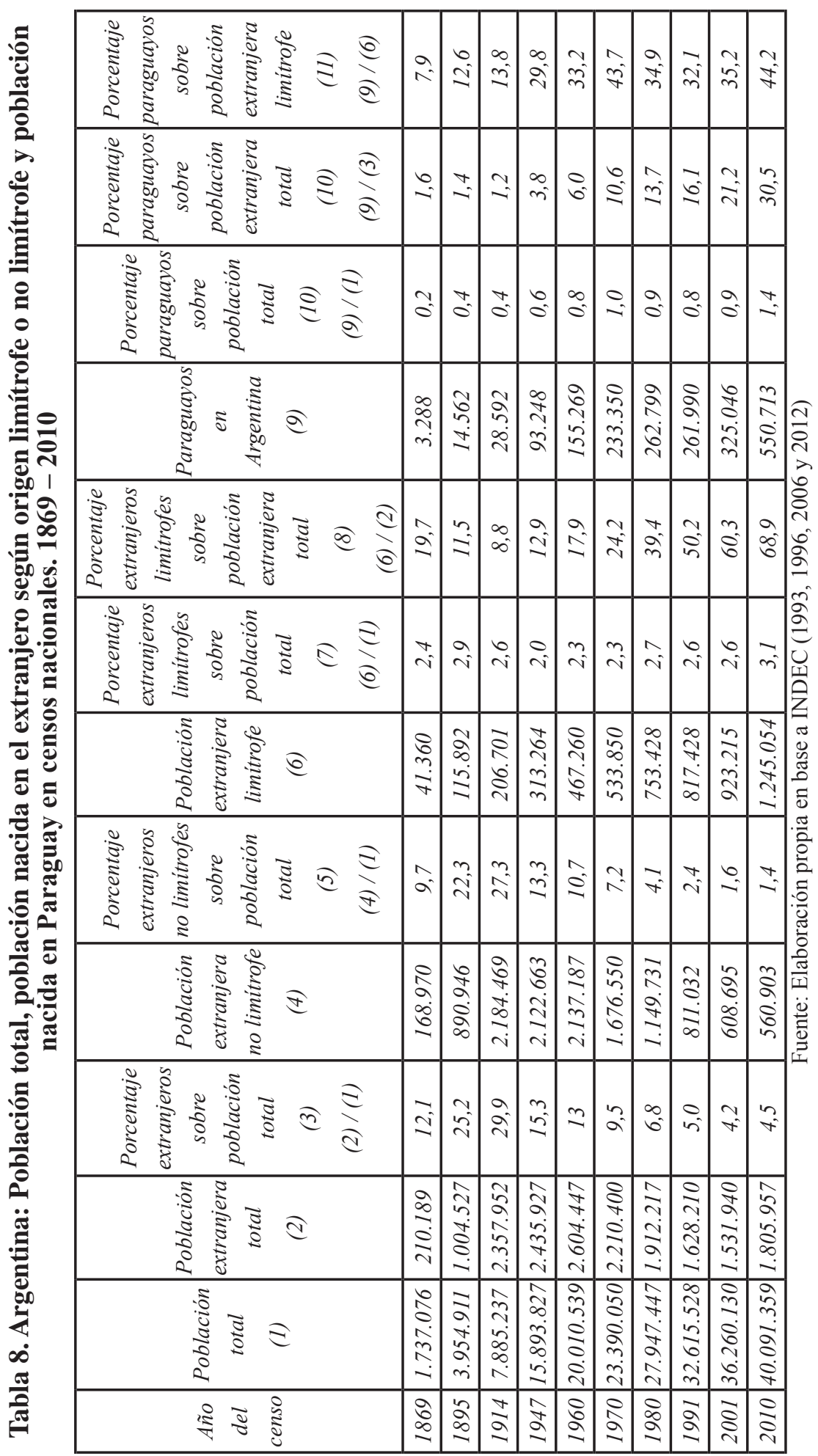

